\title{
MetaFlow|mics: Scalable and Reproducible Nextflow Pipelines for the Analysis of Microbiome Marker Data
}

\author{
Cédric Arisdakessian \\ University of Hawai'i at Mānoa, \\ Department of Information and \\ Computer Sciences \\ Honolulu, USA
}

\author{
Sean B. Cleveland \\ University of Hawai'i i at Mānoa, \\ System \\ Honolulu, USA
}

\author{
Mahdi Belcaid* \\ mahdi@hawaii.edu \\ University of Hawai'i at Mānoa, \\ Department of Information and \\ Computer Sciences \\ Honolulu, USA \\ University of Hawai'i at Mānoa, \\ Hawai'i Institute of Marine Biology \\ Honolulu, USA
}

\begin{abstract}
Computational scalability has become an important requirement for processing the massive amounts of data generated in contemporary sequencing-based experiments. The availability of large computational resources through academic, regional or national cyber-infrastructure efforts, as well as through inexpensive cloud offerings, has shifted the bottleneck, which now lies in the extensive expertise necessary to create reproducible and scalable bioinformatics pipelines and deploy them to such diverse infrastructures. We present here MetaFlow|mics, a comprehensive pipeline for the analysis of microbiome marker data using best practices and stateof-the-art cyberinfrastructure standards to ensure reproducibility. MetaFlow|mics provides seamless scalability and extensibility, allowing users to build and test their pipelines on a laptop with small datasets and to subsequently run them on large datasets on an HPC or on the Cloud with a change to a single line of code. Our framework is built on top of the Nextflow workflow management system and provides an interoperable architecture that leverages self-contained Docker and Singularity instances with all the dependencies and requirements needed to quickly deploy and use the pipeline.
\end{abstract}

\section{CCS CONCEPTS}

- Applied computing $\rightarrow$ Bioinformatics; $•$ Computing methodologies $\rightarrow$ Distributed computing methodologies.

\section{KEYWORDS}

metagenomics, microbiome, pipeline

$\overline{{ }^{*} \text { Corresponding author }}$

Permission to make digital or hard copies of part or all of this work for personal or classroom use is granted without fee provided that copies are not made or distributed for profit or commercial advantage and that copies bear this notice and the full citation on the first page. Copyrights for third-party components of this work must be honored

For all other uses, contact the owner/author(s).

PEARC '20, July 26-30, 2020, Portland, OR, USA

(C) 2020 Copyright held by the owner/author(s).

ACM ISBN 978-1-4503-6689-2/20/07.

https://doi.org/10.1145/3311790.3396664
ACM Reference Format:

Cédric Arisdakessian, Sean B. Cleveland, and Mahdi Belcaid. 2020. MetaFlow|mics: Scalable and Reproducible Nextflow Pipelines for the Analysis of Microbiome Marker Data. In Practice and Experience in Advanced Research Computing (PEARC '20), fuly 26-30, 2020, Portland, OR, USA. ACM, New York, NY, USA, 5 pages. https://doi.org/10.1145/3311790.3396664

\section{INTRODUCTION}

Microbes, which include bacteria, fungi and viruses and are collectively known as the microbiome, are an integral part of the biosphere. Multicellular organisms such as animals and plants rely extensively on microbes for carrying many critical processes such as fighting off disease, sustaining the air we breathe and the water we drink. In fact, microbes occupy almost every surface of the human body and are the most abundant organisms on Earth's landscape, with a diversity estimated at as many as 1 trillion species [7]. While the last decade has witnessed a leap in microbiome research, this field is still considered in its infancy, with mechanistic studies of the links between the microbiome and biological processes still underway.

The large datasets that are commonly produced from microbiome sequencing experiments are often overwhelming to corral and require not only advanced computational tools but also familiarity with high-performance infrastructures required to run the analyses at scale. There is a plethora of tools and pipelines for analyzing microbial data. Notable among these are Mothur[10], QIIME2[2], metaAMP [6], VSEARCH[9] and DADA2[3], which provide an extensive ecosystem of subprograms to carry out the vast majority of the steps involved in the analysis of microbiome marker data. Rather than choosing one of these pipelines, experienced users typically tailor their own custom hybrid workflows by choosing subprograms from these ecosystems to fit their exact needs. Unfortunately, the lack of smooth interoperability across these ecosystems makes designing such hybrid pipelines difficult as it requires custom fittings to connect inputs and outputs of subprograms from across ecosystems. The extensive dependencies associated with such hybrid pipelines render their deployment time-consuming for expert users and impossible for novices, particularly when deploying to new computational environments. Similarly, updating these hybrid pipelines to account for new subprograms often involves writing new scripts, making them hard to maintain and update. 
In terms of reproducibility, it has become common, and in some cases required, to publish data and scripts describing the analysis to GitHub, which has emerged as a de-facto platform for sharing data and analysis steps. However, the mere availability of data and scripts does not guarantee reproducibility as there are often platform-specific dependencies that can be complicated to configure in new environments.

Here we introduce MetaFlow|mics, a novel framework which wraps functionality from DADA2, VSEARCH and Mothur among other tools, into an easy to use a collection of pipelines for analyzing microbiome marker data. Our pipeline spans beyond an ordered list of commands (Standard Operating Procedures; or SOP) and consists of a complete framework built using standards that facilitate crossplatform portability and promotes extensibility and reproducibility.

The MetaFlow|mics framework implements three high-level tasks, a custom demultiplexing pipeline, as well as two end-to-end analysis pipelines; one for analyzing bacterial data (16S marker) and the second for use with fungi (ITS) marker data. Each of the analysis pipelines contains all the necessary tools and scripts to QA/QC the reads, and estimate the diversity both at the operational taxonomic unit level and as exact sequence variants. The analysis pipelines are implemented using Nextflow [5], $\mathrm{R}$ and Python and distributed using Docker and Singularly containerization technologies through the Docker Hub. Nextflow is a workflow management system that aims at optimizing the deployment of workflows on multiple types of platforms with minimal efforts.

In collaboration with the Center for MICROBIOME Analysis through Island Knowledge and Investigations (C-MAIKI), the Hawaii EPSCoR Ike Wai project and the Hawaii Data Science Institute, the pipelines implemented in MetaFlow|mics are also accessible through the C-MAIKI Science Gateway (https://cmaiki.its.hawaii. edu) and Ike Wai gateway[4]. These gateways provide platforms for accessing advanced high-performance computing resources and storage to support and accelerate microbiome research. By leveraging the Tapis framework, these gateways make microbial sequence analysis workflows and data easier to access, launch, track, manage and reproduce through a user-friendly web-accessible interface. The extendable application interface currently supports the MetaFlow|mics pipeline analyses and provides the tracking of all input/output data and analysis parameters to provide notifications and provenance information for every analysis run. The gateways currently offer large shared storage space with basic file handling functions including upload and download via a web browser, or Globus[1], a robust, high-speed file transfer service.

In what follows, we describe the MetaFlow|mics framework, highlight its flexibility and describe how it enhances reproducibility, scalability and simplifies monitoring.

\section{METHODS}

MetaFlow|mics groups three different microbial analysis pipelines: a custom and novel probabilistic pipeline for demultiplexing sequencing reads, a $16 \mathrm{~S}$ barcode pipeline for analyzing bacterial data, and an ITS pipeline for analyzing fungal data. The pipelines are summarized on figure 1 and further described below.

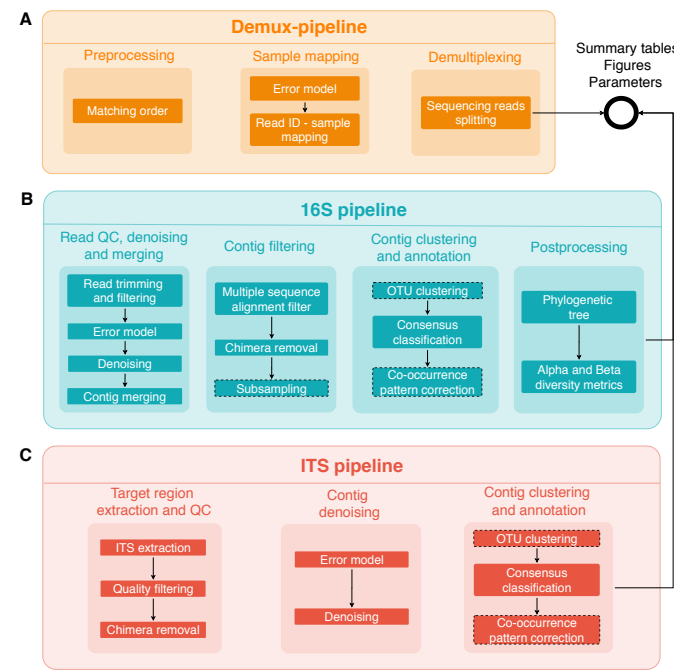

Figure 1: Overview of the three analysis pipelines steps in MetaFlow|mics. A: Demultiplexing pipeline, B: $16 \mathrm{~S}$ pipeline, C: ITS pipeline. Each box is a computation step, dotted boxes represent optional steps.

\subsection{Demultiplexing}

Modern sequencing instruments can achieve unprecedented throughput, generating terabytes of data and surpassing the minimum throughput requirements needed to sequence a single biological sample. As such, multiple biological samples are routinely combined and sequenced onto the same sequencing run. Prior to pooling the samples, a unique DNA barcode consisting of a few DNA nucleotides (A, C, G and T) is used to identify each of the samples. The DNA sequences generated by the sequencing instrument are subsequently demultiplexed, i.e., unpooled, by reading the index occurring at the beginning of each DNA sequence and placing the sequence in the appropriate sample file. In an ideal scenario, the mapping between the first few nucleotides, or bases, and the sample should consist of a simple look-up in a dictionary data-structure. However, sequencing errors, which may modify the first few bases in each sequence, render the identification of the sample of origin complex. The MetaFlow|mics demultiplexing script uses a novel probabilistic model to rescue sequences with barcode regions that do not match against any of the known barcodes. For each of the millions or billions of indices in the datasets and their associated sequencing quality scores, the probability that the index sequence was generated from any of the possible barcode sequences is computed. This can be done efficiently by partitioning the input file into many chunks of fixed size (default: 100k sequences) and processing the chunks either sequentially to facilitate checkpointing linearly or in parallel to facilitate parallelization and checkpointing of the computation. The demultiplexing pipeline is written in Nextflow's domain-specific language, allowing for seamless parallelizing of the pipeline regardless of the underlying infrastructure.

The pipeline produces a detailed account of the number of reads assigned to each sample and further breaks down the results by indicating whether the index matches the barcode perfectly, as well 
as the number of indexes containing errors. The pipeline provides a visualization of the overall read quality distribution as well as the log-transformed distribution of the number of sequences in the total samples (figure 3.A). This visualization is used later in the analysis to set parameters such as the truncation length and the subsampling threshold (in the $16 \mathrm{~S}$ pipeline) but also for identifying aberrant demultiplexing patterns, which may require immediate attention.

\subsection{Microbial analysis pipelines (16S and ITS markers)}

To study the microbial composition in a given environment, a very popular approach consists in comparing the relative amounts of different taxa found in the collected samples. Microbial composition and abundance in a given environment are cornerstone features in microbiology. These features are typically measured by sequencing a "marker gene" that is highly conserved between individuals of a species and sufficiently variable across species, which is important to minimize false positives. The $16 \mathrm{~S}$ and ITS markers are respectively the two most commonly used markers in bacterial and fungal projects $[8,11,12]$. Although the analysis of these two markers involves similar high-level processing steps, these markers have very different properties and their analysis requires independent programs and pipelines.

The $16 \mathrm{~S}$ data analysis pipeline encompasses 14 distinct steps (figure 1.B), which can be divided into four distinct stages. In the first stage, sequencing reads are trimmed, filtered and denoised to limit the impact of sequencing errors on the downstream analysis. In the second stage, sequencing reads are merged into longer sequences, and outlier sequences that deviate from the expected fragment length distribution are filtered out. In the third stage, contigs are clustered into OTUs, or clusters grouping taxonomically related sequences, and annotated at various taxonomic levels. The last stage consists of producing figures and tables summarizing the results and facilitating troubleshooting and downstream analysis.

The ITS data analysis pipeline is comprised of 11 different steps (figure 1.C), which are conceptually similar to those described for the $16 \mathrm{~S}$ pipeline but which take into account molecular differences between the two markers. For instance,unlike the $16 \mathrm{~S}$ marker, the ITS marker is known to have a variable length. This requires a custom algorithm to identify the boundaries of the ITS marker in the sequencing reads prior to proceeding to the $\mathrm{QC} / \mathrm{QA}$ stage.

\subsection{Pipeline implementation}

The analysis of contemporary metagenomic experiments, which can contain millions of reads, requires significant computational resources. Unfortunately, the source of such resources in microbiome experiments often differs across projects. A major objective of our pipeline is to facilitate its deployment and parallelization on high performance environments such as High Performance Computing (HPCs) clusters or cloud computing services. All of these pipelines were tested on multiple devices, including local computers, remote servers, the University of Hawai' $i$ at Mānoa HPC cluster and Google Cloud.

2.3.1 Reproducibility. Given an analysis pipeline and a dataset, the main cause of variation in the results is the version of the software and the Operating System (OS) in use. For instance, in a recent research paper published in Organic Letters, Neupane et al. showed that a popular Python script ("Willoughby-Hoye") was OS-dependent and that published analyses based on this script were in some cases erroneous [Neupane et al. 2019]. MetaFlow|mics addresses reproducibility in general and lack of consistency across environments (OS, architecture, program versions, etc.) in particular by leveraging containerized computing. Details of the computing environment required to run each of the pipelines is described in Dockerfiles that bundle all necessary deployment information, such as operating system type and version and software versions. The Dockerfiles are then used to faithfully reproduce any environment as a standalone container. Additionally, the pipelines are configured to generate a listing of all the commands executed during an analysis, providing the users with an additional transcript that can be shared and used to reproduce an analysis. For backward compatibility considerations, previous pipeline versions can be accessed on GitHub, providing the user with a way to switch to previous run settings if needed.

2.3.2 Scalability. Many of the modules used in the pipeline process each sample independently, which makes parallelization effective and resource-efficient. Nextflow streamlines parallelization by automatically transferring data between machines, running independent tasks in parallel (or linearly if resources are limited) and collecting results. The pipeline provides preset configuration files to facilitate deployment on HPC clusters (SLURM, SGE among others) and cloud environments (Google Cloud). The description of required resources, such as queues names in an HPC environment or machine type in Google Cloud can be specified by the user independently for each task of the pipeline or assigned automatically in some cases based on predetermined default values. Such low-granularity in resource allocation can speed up the runtime and minimize cost when using a cloud computing service. Finally, MetaFlow|mics can automatically scale up if a process unexpectedly exceeds the resources it was allocated. For example, if a process exceeds its wall time or uses too much memory, it will be re-submitted with larger wall time or memory requirements.

2.3.3 Flexibility. As discussed above, the implementation environment is not coupled to the source code implementing the analysis logic. As shown on figure 2, the software dependencies and environment are defined with Docker and both Nextflow and MetaFlow|mics can be bootstrapped from Docker Hub. The pipelines also support Singularity containerization technology and can therefore run securely in a multi-user environment where user permissions are limited. Thus, the minimal user intervention consists in installing Docker (or Singularity) on the machine. These frameworks are typically already available on most shared systems like HPC clusters and cloud services.

The required configuration that users need to provide is minimal and consists of platform-specific information such as the account and project names for billing under Google Cloud or the scheduler and queues/partitions to use in an HPC environment. The configuration only needs to be defined once per infrastructure instance. Once the correct information is supplied, very little work is required, regardless of the underlying infrastructure. To maximize flexibility, 


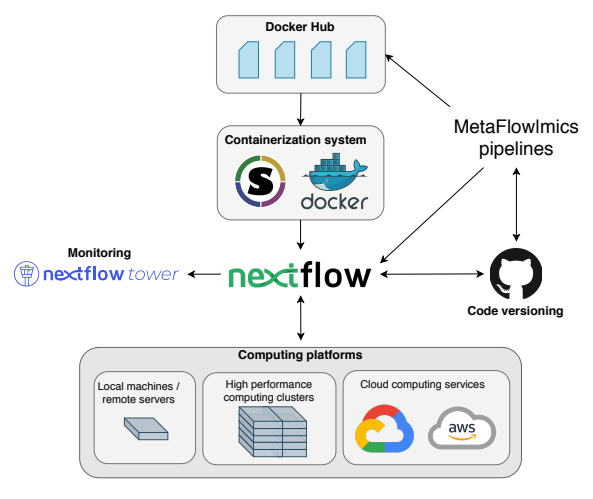

Figure 2: MetaFlow|mics workflow: MetaFlow|mics source code is hosted on GitHub, and required containers were built and uploaded on Docker Hub. Nextflow builds an abstraction layer on any configured platforms, and can run any of the pipeline using the provided docker images. Job monitoring is then easily achieved using Nextflow Tower.

switching between platforms can be achieved by changing a single command-line argument:
A

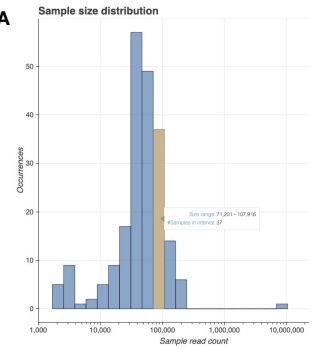

C

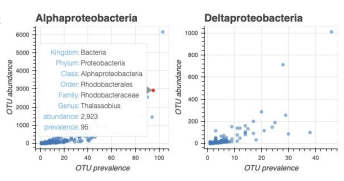

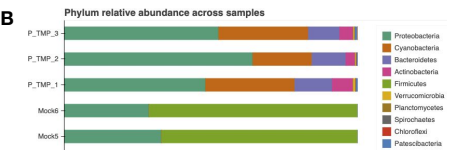

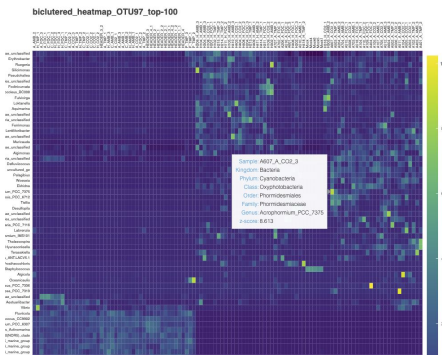

Figure 3: Pipelines summary figures. Each figure is interactive and displays additional information about the data points. A: Log sample size distribution. B: Stacked barplot of phyla proportions in each sample. C: Scatter plot of OTU prevalence vs abundance for the Alphaproteobacteria and Deltaproteobacteria classes. D: Heatmap of the top 100 OTU by samples. Both rows and columns are clustered (hierarchical bi-clustering) to identify patterns.

nextflow run Pipeline-16S --reads "/data/*_R $\{1,2\} *$.fastq" -profile local

nextflow run Pipeline-16S --reads "/data/*_R\{1,2\}*.fastq" -profile gcp

In a distributed environment, multiple processes can use multiple machines shared by multiple users. As a result, it is very common to encounter machine failures which would cause many pipelines to abruptly stop. While also subject to external failures, MetaFlow|mics leverage Nexflow's check-pointing capabilities and can resume interrupted processes starting from the most recent checkpoint.

2.3.4 Monitoring. The pipelines can generate information-dense outputs, which can be difficult to parse and interpret; particularly to identify anomalies in the execution of the pipeline, or to monitor the run progress. MetaFlow|mics mitigates this by providing two types of execution outputs. The first type of output consists of a set of task-specific data visualizations (heatmaps, scatter, bar and box plots) that graphically describe the results (see figure 3). The second type of output consists of a table that summarizes the number of sequences left in each sample after each step of a pipeline. This table is ideal for detecting odd samples, incorrect settings or processing errors. For instance, when sequencing long DNA regions, the $16 \mathrm{~S}$ pipeline will filter out many sequences if the trimming parameter is not correctly set. This becomes obvious when looking at the number of remaining sequences after the read merging step.

Furthermore, Nextflow can be paired with Nextflow Tower (https: //tower.nf) an open-source, hosted solution that provides real-time monitoring of each pipeline's execution. This is especially useful in distributed environments where monitoring the executions of all the processes often requires custom code. In addition to realtime updates, Nextflow Tower provides useful statistics describing the pipeline tasks, including a breakdown of resource usage (CPU, memory, time, IO, etc.). Configuring and using Nextflow Tower only requires adding an environment variable with the Nextflow Tower account information (i.e. a token) to the pipeline configuration and using "-with-tower" flag when executing the pipeline.
The monitoring information is then directly available through the Nextflow Tower website.

\section{DISCUSSION AND CONCLUSION}

Pipelines are essential in bioinformatics data analysis, particularly in projects where large datasets and multiple analysis steps are involved. Implementations of microbiome data analysis pipelines may differ for reasons such as the implementer's experience, their individualistic software preferences, and the computational resources at hand. As such, pipelines are often project-specific and lack the seamless interoperability necessary for code reuse. In fact, expert users may find it easier to write a new pipeline rather than adapt one from a different project. In contrast, inexperienced users often lack the skills needed to modify the code to fit their needs. The expansive set of tools available for analyzing microbiome data, while a boon for productivity, further complicates code portability as swapping a program in a pipeline often requires patching the code to account for the operational differences. MetaFlow|mics handles this issue by wrapping the specifics of each program it uses in computation processes; those are tasks, or units, of execution in the Nextflow framework. Thus, one can modify a task in the pipeline without changing any of the upstream or downstream tasks. Also, defining resources' requirements at the task level decouples configuration from data analysis logic, which, in turn, improves code portability and scalability. Additionally, the task-specific resource allocation, as opposed to allocating resources at the pipeline level, optimizes resource usage since each task is getting only what it needs. The drawback, in this case, is the additional time required to assign resources for each task. To simplify configuration, Nextflow allows users to create aliases for recurring settings and reuse those 
across tasks. MetaFlow|mics also provides out-of-the-box configuration aliases for common execution environments, such as servers, Google Cloud, and HPC environments.

The pleasantly parallel nature of most tasks in microbiome data analysis facilitates scalability since chunks of a large file can be processed in parallel, and fault-tolerance since failures do not affect successfully processed data chunks.

Lastly, it is a common misconception that pipelines are a panacea for reproducibility. This is not only inaccurate but also misleading since it provides a false sense of confidence in the results. In fact, substantial variations in data analysis can arise due to differences in OS and software versions used. By implementing all the tasks as Docker and Singularity instances, MetaFlow|mics enforces reproducibility and transparency without compromising usability.

In summary, MetaFlow|mics is a robust and easy to use pipeline that leverages best practices and state-of-the-art cyberinfrastructure standards to provide seamless scalability, interoperability, and extensibility.

\section{FUTURE WORK}

Future work will include adding additional configurations for XSEDE resources to enable easier deployment of the pipelines to some of these systems. Further, the integration with Jetstream's OpenStack API for access on-demand clouds resources for MetaFlow|mics will be pursued.

\section{SOFTWARE AVAILABILITY}

The code of the pipeline is available on GitHub at https://github. com/hawaiidatascience/metaflowmics and the documentation was compiled and deposited on ReadTheDocs at https://metagenomicspipelines.readthedocs.io/en/latest

\section{ACKNOWLEDGMENTS}

This material is based upon work supported by the National Science Foundation Office of Advanced CyberInfrastructure[grant numbers 1931439 and 1931575], RII Track 1: 'Ike Wai Securing Hawai'i's Water Future NSF OIA \#1557349, Division of Ocean Sciences \#1636402, Collaborative Research: SS2-SSI: The Agave Platform: An Open Science-As-A-Service Cloud Platform for Reproducible Science NSF ACI \#1450413.

\section{REFERENCES}

[1] William Allcock, John Bresnahan, Rajkumar Kettimuthu, and Michael Link. 2005 The Globus striped GridFTP framework and server. In SC'05: Proceedings of the 2005 ACM/IEEE conference on Supercomputing. IEEE, 54-54.

[2] Evan Bolyen, Jai Ram Rideout, Matthew R Dillon, Nicholas A Bokulich, Christian Abnet, Gabriel A Al-Ghalith, Harriet Alexander, Eric J Alm, Manimozhiyan Arumugam, Francesco Asnicar, and others. 2018. QIIME 2: Reproducible, interactive, scalable, and extensible microbiome data science. Technical Report. PeerJ Preprints.

[3] Benjamin J Callahan, Paul J McMurdie, Michael J Rosen, Andrew W Han, Amy Jo A Johnson, and Susan P Holmes. 2016. DADA2: high-resolution sample inference from Illumina amplicon data. Nature methods 13, 7 (2016), 581.

[4] Sean B Cleveland, Rion Dooley, David Perry, Joe Stubbs, John M Fonner, and Gwen A Jacobs. 2018. Building science gateway infrastructure in the middle of the pacific and beyond: experiences using the agave deployer and agave platform to build science gateways. In Proceedings of the Practice and Experience on Advanced Research Computing. 1-8.

[5] Paolo Di Tommaso, Maria Chatzou, Evan W Floden, Pablo Prieto Barja, Emilio Palumbo, and Cedric Notredame. 2017. Nextflow enables reproducible computational workflows. Nature biotechnology 35, 4 (2017), 316-319.
[6] Xiaoli Dong, Manuel Kleiner, Christine E Sharp, Erin Thorson, Carmen Li, Dan Liu, and Marc Strous. 2017. Fast and simple analysis of MiSeq amplicon sequencing data with MetaAmp. Frontiers in microbiology 8 (2017), 1461.

[7] Kenneth J Locey and Jay T Lennon. 2016. Scaling laws predict global microbial diversity. Proceedings of the National Academy of Sciences 113, 21 (2016), 59705975.

[8] Linda Medlin, Hille J Elwood, Shawn Stickel, and Mitchell L Sogin. 1988. The characterization of enzymatically amplified eukaryotic 16S-like rRNA-coding regions. Gene 71, 2 (1988), 491-499.

[9] Torbjørn Rognes, Tomáš Flouri, Ben Nichols, Christopher Quince, and Frédéric Mahé. 2016. VSEARCH: a versatile open source tool for metagenomics. Peerf 4 (2016), e2584.

[10] Patrick D Schloss, Sarah L Westcott, Thomas Ryabin, Justine R Hall, Martin Hartmann, Emily B Hollister, Ryan A Lesniewski, Brian B Oakley, Donovan H Parks, Courtney J Robinson, and others. 2009. Introducing mothur: open-source, platform-independent, community-supported software for describing and comparing microbial communities. Appl. Environ. Microbiol. 75, 23 (2009), 7537-7541.

[11] Thomas J White, Thomas Bruns, SJWT Lee, John Taylor, et al. 1990. Amplification and direct sequencing of fungal ribosomal RNA genes for phylogenetics. PCR protocols: a guide to methods and applications 18, 1 (1990), 315-322.

[12] Carl R Woese. 1987. Bacterial evolution. Microbiological reviews 51, 2 (1987), 221. 